Byung Gyun Kang · Dong Yeol Oh

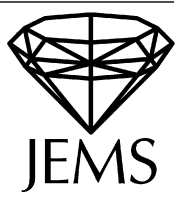

\title{
Formal power series rings over a $\pi$-domain
}

Received December 28, 2007 and in revised form August 19, 2008

\begin{abstract}
Let $R$ be an integral domain, $\mathcal{X}$ be a set of indeterminates over $R$, and $R[[\mathcal{X}]]_{3}$ be the full ring of formal power series in $\mathcal{X}$ over $R$. We show that the Picard group of $R[[\mathcal{X}]]_{3}$ is isomorphic to the Picard group of $R$. An integral domain is called a $\pi$-domain if every principal ideal is a product of prime ideals. An integral domain is a $\pi$-domain if and only if it is a Krull domain that is locally a unique factorization domain. We show that $R[[\mathcal{X}]]_{3}$ is a $\pi$-domain if $R\left[\left[X_{1}, \ldots, X_{n}\right]\right]$ is a $\pi$-domain for every $n \geq 1$. In particular, $R[[\mathcal{X}]]_{3}$ is a $\pi$-domain if $R$ is a Noetherian regular domain. We extend these results to rings with zero-divisors. A commutative ring $R$ with identity is called a $\pi$-ring if every principal ideal is a product of prime ideals. We show that $R[[\mathcal{X}]]_{3}$ is a $\pi$-ring if $R$ is a Noetherian regular ring.
\end{abstract}

Keywords. Krull domain, $\pi$-domain, unique factorization domain, formal power series ring, invertible ideal, class group, Picard group

\section{Introduction}

The question whether the power series ring over a unique factorization domain (UFD) is a UFD had remained open for a long time until Samuel constructed a Noetherian counterexample in [17]. However, there do exist UFDs $R$ such that $R\left[\left[X_{1}, \ldots, X_{n}\right]\right]$ is a UFD for every $n$, e.g., regular UFDs, and more specifically principal ideal domains. All of these examples are Noetherian. Non-Noetherian examples were constructed by Deckard [7], Cashwell and Everette [5] as well as Nishimura [16] (see also Deckard and Durst [8]) exploring power series rings in an infinite number of variables. Recall that in the case of an infinite number of variables, there are several types of power series rings. Let $\mathcal{X}=\left\{X_{\lambda}\right\}_{\lambda \in \Lambda}$ be a set of indeterminates over $R$, and $S$ be the weak direct sum of the additive abelian semigroup $\mathbb{N}$ with itself $|\Lambda|$ times, where $\mathbb{N}$ is the set of nonnegative integers. Following the notation and definition in [10,11], the full ring offormal power series in $\mathcal{X}$ over $R$ is defined to be the set of all functions $f: S \rightarrow R$, where $(f+g)(s)=f(s)+g(s)$ and $(f g)(s)=\sum_{t+u=s} f(t) g(u)$ for any $s \in S$, the notation $\sum_{t+u=s}$ indicating that the sum is taken over all ordered pairs $(t, u)$ of elements of $S$ with sum $s$. The full ring of formal power series in $\mathcal{X}$ over $R$ is denoted by $R[[\mathcal{X}]]_{3}$ while $R[[\mathcal{X}]]_{2}$ denotes the $(\mathcal{X})$ adic completion of the polynomial ring $R[\mathcal{X}]$. The aforementioned authors showed that

B. G. Kang, D. Y. Oh: Department of Mathematics, Pohang University of Science and Technology, Pohang 790-784, The Republic of Korea; e-mail: bgkang@ postech.ac.kr, dyoh@ postech.ac.kr

Mathematics Subject Classification (2000): Primary 13J05; Secondary 13C20, 13A15 
for an infinite set $\mathcal{X}$ of indeterminates over a domain $R, R[[\mathcal{X}]]_{3}$ is a UFD as long as $R\left[\left[X_{1}, \ldots, X_{n}\right]\right]$ is a UFD for every $n$. Thus $R[[\mathcal{X}]]_{3}$ is a non-Noetherian UFD such that its arbitrary power series extensions are UFDs provided that $R\left[\left[X_{1}, \ldots, X_{n}\right]\right]$ is a UFD for every $n$. As for the divisor class groups, Samuel's example [17] shows that $\mathrm{Cl}(R[[X]]) \nsubseteq \mathrm{Cl}(R)$ in general. Indeed, $\mathrm{Cl}(R[[X]]) \cong \mathrm{Cl}(R) \oplus \mathrm{Cl}\left(R[[X]]_{R^{*}}\right)$ for a Noetherian Krull domain $R$. However, for the Picard group of an integral domain $R$, $\operatorname{Pic}(R[[X]]) \cong \operatorname{Pic}(R)$. From this fact, it follows that for a UFD $R, R[[X]]$ is a UFD if and only if $R[[X]]_{R^{*}}$ is a UFD if and only if $R[[X]]$ is a $\pi$-domain.

These results motivate us to investigate power series extensions of a $\pi$-domain. An integral domain is called a $\pi$-domain if every principal ideal is a product of prime ideals. Recall that an integral domain is a $\pi$-domain if and only if it is a Krull domain that is locally a UFD. Thus $\pi$-domains lie between Krull domains and UFDs. Gilmer [10] showed that for a Krull domain $R, R[[\mathcal{X}]]_{3}$ is a Krull domain. So it is natural to ask if $R[[\mathcal{X}]]_{3}$ is a $\pi$-domain when $R$ is a $\pi$-domain such that $R\left[\left[X_{1}, \ldots, X_{n}\right]\right]$ is a $\pi$-domain for each $n \geq 1$. The main purpose of this paper is to answer this question. To help the readers better understand $\pi$-domains, we quote a couple of their characterizations. An integral domain is a $\pi$-domain if and only if every t-ideal is an invertible ideal if and only if every principal ideal is a product of prime ideals if and only if every nonzero prime ideal contains an invertible prime ideal [1, 2, 12]. The most well-known examples of $\pi$ domains are Noetherian regular domains. A regular local ring $R$ is a UFD (the AuslanderBuchsbaum theorem [3]), each $R\left[\left[X_{1}, \ldots, X_{n}\right]\right]$ is a regular local ring, and in this case we have already mentioned that its arbitrary power series extension $R[[\mathcal{X}]]_{3}$ is also a UFD. However, nothing is known about the global case, i.e., about $R[[\mathcal{X}]]_{3}$ for a Noetherian regular domain $R$. One of our main results is that $R[[\mathcal{X}]]_{3}$ is a $\pi$-domain for a Noetherian regular domain $R$. This provides examples of non-Noetherian $\pi$-domains whose full rings of formal power series extensions are also $\pi$-domains. In fact, for an integral domain $R$, we prove the stronger result that $R[[\mathcal{X}]]_{3}$ is a $\pi$-domain if and only if $R\left[\left[X_{1}, \ldots, X_{n}\right]\right]$ is a $\pi$-domain for every $n \geq 1$. In the process, we prove that $\operatorname{Pic}\left(R[[\mathcal{X}]]_{3}\right) \cong \operatorname{Pic}(R)$ for an integral domain $R$. We extend the results on integral domains to rings with zero-divisors. A commutative ring $R$ with identity is called a $\pi$-ring if every principal ideal is a product of prime ideals. We show that $R[[\mathcal{X}]]_{3}$ is a $\pi$-ring if $R$ is a Noetherian regular ring.

\section{Preliminaries}

In this section we introduce concepts and basic properties of power series rings which are needed in what follows. Our general reference for power series rings is Brewer's [4]. Throughout this paper (except in Section 5), $R$ will be an integral domain and $\mathcal{X}$ will stand for a set of indeterminates. Note that an element $f$ of $R[[\mathcal{X}]]_{3}$ can be written as the formal sum $f=\sum c\left(a_{i_{1}}, \ldots, a_{i_{n}}\right) X_{i_{1}}^{a_{i_{1}}} \ldots X_{i_{n}}^{a_{i_{n}}}$, where $\left\{X_{i_{1}}, \ldots, X_{i_{n}}\right\}$ ranges over the finite subsets of $\mathcal{X}, a_{i_{j}}$ 's are nonnegative integers, and $c\left(a_{i_{1}}, \ldots, a_{i_{n}}\right)$ belongs to $R$. For a finite subset $\mathcal{F}$ of $\mathcal{X}$, define a map $\pi_{\mathcal{F}}$ from $R[[\mathcal{X}]]_{3}$ onto $R[[\mathcal{F}]]$ by evaluating at $X_{i}=0$ for all $X_{i} \notin \mathcal{F}$. Clearly the map is a ring epimorphism, called the canonical projection with respect to $\mathcal{F}$. For each $\alpha \in R[[\mathcal{X}]]_{3}$, define the $\mathcal{F}$-projection of $\alpha$ to be the element 
$\mathcal{F}(\alpha)$, which is the image of $\alpha$ under the canonical projection with respect to $\mathcal{F}$. For a subset $I$ of $R[[\mathcal{X}]]_{3}$, we denote by $\mathcal{F}(I)$ the set of all $\mathcal{F}$-projections of elements of $I$. In particular, if $\mathcal{F}=\emptyset$, then the $\emptyset$-projections of $\alpha$ and $I$ will be denoted by $\alpha(0)$ and $I_{0}:=\emptyset(I)$, respectively.

Given a finite subset $\mathcal{F}$ of $\mathcal{X}$, we define a sequence over $\mathcal{F}$ to be a function which assigns to every finite subset $\mathcal{K}$ of $\mathcal{X}$ containing $\mathcal{F}$ a definite element $\alpha_{\mathcal{K}}$ of $R[[\mathcal{K}]]$. A sequence over $\mathcal{F}$ is called projective if $\mathcal{K}\left(\alpha_{\mathcal{L}}\right)=\alpha_{\mathcal{K}}$ whenever $\mathcal{F} \subseteq \mathcal{K} \subseteq \mathcal{L}$. A sequence over $\mathcal{F}$ is called associative if $\mathcal{K}\left(\alpha_{\mathcal{L}}\right) \sim \alpha_{\mathcal{K}}$ in $R[[\mathcal{K}]]$ whenever $\mathcal{F} \subseteq \mathcal{K} \subseteq \mathcal{L}$. Here $\beta \sim \gamma$ means $\beta=\gamma \nu$ for some unit $\nu$ of the indicated domain.

We quote useful results from [5], adding a couple of minor new results.

Theorem 2.1. Let $\mathcal{X}$ be a set of indeterminates, and $\mathcal{F}$ be a finite subset of $\mathcal{X}$. Then, for $\alpha, \beta \in R[[\mathcal{X}]]_{3}$,

(1) $\mathcal{F}(\alpha \beta)=\mathcal{F}(\alpha) \mathcal{F}(\beta)$ and $\mathcal{F}(\alpha+\beta)=\mathcal{F}(\alpha)+\mathcal{F}(\beta)$,

(2) $\mathcal{F}(\mathcal{K}(\alpha))=\mathcal{F}(\alpha)$ if $\mathcal{K}$ is a finite subset of $\mathcal{X}$ containing $\mathcal{F}$,

(3) if $\mathcal{F}(\alpha)$ is a unit of $R[[\mathcal{F}]]$, then $\alpha$ is a unit of $R[[\mathcal{X}]]_{3}$,

(4) if $\mathcal{K}(\alpha)=\mathcal{K}(\beta)$ for every finite subset $\mathcal{K} \supseteq \mathcal{F}$ of $\mathcal{X}$, then $\alpha=\beta$,

(5) every projective sequence $\left\{\alpha_{\mathcal{K}}\right\}$ over $\mathcal{F}$ is of the form $\alpha_{\mathcal{K}}=\mathcal{K}(\alpha)$ for some $\alpha \in$ $R[[\mathcal{X}]]_{3}$

(6) for every associative sequence $\left\{\alpha_{\mathcal{K}}\right\}$ over $\mathcal{F}$, there exists an element $\alpha \in R[[\mathcal{X}]]_{3}$ such that $\mathcal{K}(\alpha) \sim \alpha_{\mathcal{K}}$ in $R[[\mathcal{K}]]$ for every finite subset $\mathcal{K} \supseteq \mathcal{F}$ of $\mathcal{X}$,

(7) if I and $J$ are ideals of $R[[\mathcal{X}]]_{3}$, then $\mathcal{F}(I)$ and $\mathcal{F}(J)$ are ideals of $R[[\mathcal{F}]]$; moreover, $\mathcal{F}(I+J)=\mathcal{F}(I)+\mathcal{F}(J)$ and $\mathcal{F}(I J)=\mathcal{F}(I) \mathcal{F}(J)$

(8) for an ideal $I$ of $R[[\mathcal{X}]]_{3}$, we have $\mathcal{F}(\mathcal{K}(I))=\mathcal{F}(I)$ if $\mathcal{K}$ is a finite subset of $\mathcal{X}$ containing $\mathcal{F}$.

Proof. (1)-(6) are proved in [5]. From (1), (2), and the fact that the projection map is a ring epimorphism, (7) and (8) follow.

We briefly review the definition of a divisorial ideal and relations between a Krull domain and a $\pi$-domains.

Let $R$ be an integral domain with quotient field $K$. By a fractional ideal $F$ we mean an $R$-submodule of $K$. By $F^{-1}$ (the inverse of $F$ ) we mean the set of all $x$ in $K$ with $x F \subseteq R$. Note that $F^{-1}$ is also a fractional ideal. We say that $F$ is invertible if $F F^{-1}=R$. For a fractional ideal $F$, we denote by $F_{v}$ the fractional ideal $\left(F^{-1}\right)^{-1}$. The fractional ideal $\sum\left\{I_{v} \mid I\right.$ is a finitely generated fractional ideal contained in $\left.F\right\}$ is denoted by $F_{t}$. If $F=F_{v}$, then $F$ is called a divisorial ideal. If $F=F_{t}$, then $F$ is called a $t$-ideal. It is well known that every divisorial ideal of a Krull domain $R$ is a $v$-product of minimal prime ideals of $R$-namely, for a divisorial ideal $I, I=\left(P_{1} \ldots P_{k}\right)_{v}$, where $P_{i}$ 's are (not necessarily distinct) minimal prime ideals of $R$. We refer the readers to [9, 11, 13] for more information on divisorial ideals.

An integral domain in which every principal ideal is a product of finitely many prime ideals is called a $\pi$-domain, which is a generalization of the unique factorization domain (UFD). The following is a relation between Krull domains and $\pi$-domains. For a proof, the readers are referred to [1, 2, 9, 11, 12, 14]. 
Theorem 2.2. The following are equivalent for a domain $R$ :

(1) $R$ is a $\pi$-domain.

(2) $R_{M}$ is a UFD for each maximal ideal $M$ of $R$, and every minimal prime ideal of $R$ is finitely generated.

(3) $R$ is a Krull domain, and every minimal prime ideal of $R$ is invertible.

(4) $R$ is a Krull domain, and the product of any finite number of divisorial ideals is a divisorial ideal.

(5) Every divisorial ideal is a finite product of prime ideals.

(6) Every $t$-ideal is a finite product of prime ideals.

(7) Every $t$-ideal is invertible.

If $R$ is a $\pi$-domain, then it follows from Theorem 2.2 that every divisorial ideal of $R$ is a product of a finite number of minimal prime ideals of $R$-namely, for a divisorial ideal $I$ of $R$,

$$
I=P_{1}^{n_{1}} \cdots P_{k}^{n_{k}},
$$

where $P_{i}$ 's are distinct minimal prime ideals of $R$.

\section{Invertible ideals of a power series ring}

In this section, we investigate a relation between invertible ideals of an integral domain and those of its power series ring. Recall that the $\emptyset$-projection of an ideal $I$ of a power series ring is denoted by $I_{0}:=\emptyset(I)$, which is the same as the set of all the constant terms of elements in $I$.

Lemma 3.1. Let $\Phi: D \rightarrow R$ be a ring epimorphism between integral domains. If $I$ is an invertible ideal of $D$ and $\Phi(I) \neq(0)$, then $\Phi(I)$ is an invertible ideal of $R$.

Proof. Choose $0 \neq a \in I$ such that $\Phi(a) \neq 0$. Then $a D=I\left(a I^{-1}\right)$ and $a I^{-1} \subseteq D$. We have

$$
\Phi(a) R=\Phi(a D)=\Phi\left(I\left(a I^{-1}\right)\right)=\Phi(I) \Phi\left(a I^{-1}\right) .
$$

So $\Phi(I)$ is an invertible ideal of $R$.

The next result is well known. For easy reference, we include its proof.

Theorem 3.2. Let $R$ be an integral domain with quotient field $K$, and $I$ be an invertible ideal of $R[[X]]$, where $X$ is an indeterminate over $R$. Then

(i) if $I_{0} \neq(0)$, then $I_{0}$ is also an invertible ideal of $R$,

(ii) there exists an invertible ideal $I^{\prime}$ of $R$ such that $I=f I^{\prime}[[X]]$, where $f \in K[[X]]$; moreover, if $I_{0} \neq(0)$, then we can take $I_{0}$ for $I^{\prime}$. 
Proof. Choose an integer $n \geq 0$ such that $I \subseteq\left(X^{n}\right)$ and $I \nsubseteq\left(X^{n+1}\right)$. Put $J=X^{-n} I$. Then $J$ is an invertible ideal of $R[[X]]$ such that $J_{0} \neq(0)$. Therefore it suffices to show that if $I$ is an invertible ideal of $R[[X]]$ such that $I_{0} \neq(0)$, then $I_{0}$ is invertible and $I=f I_{0}[[X]]$ for some $f \in K[[X]]$.

By Lemma 3.1, $I_{0}$ is an invertible ideal of $R$. Put $J=I\left(I_{0}^{-1}[[X]]\right) a$, where $0 \neq a$ $\in I_{0}$. Since $a \in I_{0}, J$ is an ideal of $R[[X]]$. Applying the $\emptyset$-projection of $J$, we have

$$
J_{0}=\emptyset(J)=\emptyset\left(I\left(I_{0}^{-1}[[X]]\right) a\right)=\emptyset(I) \emptyset\left(I_{0}^{-1}[[X]]\right) \emptyset(a)=I_{0} I_{0}^{-1} a=a R .
$$

Since $a \in J_{0}$, we can choose an element $j \in J$ such that $j(0)=a$. Thus $J_{0}=j(0) R$. Since for each $g \in J, g=g(0)+X g_{1}$, where $g_{1} \in R[[X]]$, we have $g(0) \in J_{0}$. Hence $J \subseteq J_{0}+X R[[X]]=j(0) R+X R[[X]] \subseteq j R[[X]]+X R[[X]]$. If $g \in J$, then $g=j h+X g_{2}$, where $h, g_{2} \in R[[X]]$. Since $g, j \in J$, we have $g_{2} \in(J: X)$. Therefore, $J=j R[[X]]+X(J: X)$.

We claim that $J=(J: X)$. If $g \in(J: X)$, then $X g \in J=I\left(I_{0}^{-1}[[X]]\right) a$. Thus $X g I_{0}[[X]] \subseteq a I$. Since $a I$ is an invertible ideal of $R[[X]]$, we have $X g I_{0}[[X]]=(a I) I^{\prime}$ for some ideal $I^{\prime}$ of $R[[X]]$. Note that $a I \nsubseteq(X)$ because $I_{0}=\emptyset(I) \neq(0)$. Since $(X)$ is an invertible prime ideal, we have $I^{\prime} \subseteq(X)$, i.e., $I^{\prime}=X I^{\prime \prime}$ for some ideal $I^{\prime \prime}$ of $R[[X]]$. Hence $X g I_{0}[[X]]=(a I) I^{\prime}=a X I I^{\prime \prime}$. By cancellation, we have $g I_{0}[[X]]=(a I) I^{\prime \prime}$. Therefore, $g \in(a I) I_{0}^{-1}[[X]] I^{\prime \prime}=J I^{\prime \prime} \subseteq J$. Thus the claim is proved.

Since $J=(J: X)$, we have $J=j R[[X]]+X J$, where $j \in J$. Note that since $J$ is invertible, $J$ is a finitely generated $R[[X]]$-module. Since $X$ is in the Jacobson radical of $R[[X]]$, by the Nakayama lemma, we have $J=j R[[X]]=I\left(I_{0}^{-1}[[X]]\right) a$. Therefore, $I=a^{-1} j I_{0}[[X]]=f I_{0}[[X]]$, where $f=a^{-1} j \in K[[X]]$.

We generalize Theorem 3.2 to a finite number of indeterminates.

Corollary 3.3. Let $R$ be an integral domain with quotient field $K$, and $I$ be an invertible ideal of $R[[\mathcal{F}]]$, where $\mathcal{F}$ is a finite set of indeterminates over $R$. Then there exists an invertible ideal $I^{\prime}$ of $R$ such that

$$
I=f I^{\prime}[[\mathcal{F}]] \quad \text { for some } f \in K[[\mathcal{F}]] .
$$

Moreover, if $\emptyset(I)=I_{0} \neq(0)$, then $I=f I_{0}[[\mathcal{F}]]$ for some $f \in K[[\mathcal{F}]]$.

In a $\pi$-domain, every principal ideal is a product of prime ideals instead of principal prime ideals. So we have to consider a projective sequence of ideals instead of elements.

Definition 3.4. Let $R$ be an integral domain and $\mathcal{X}$ be a set of indeterminates over $R$. Let $\mathcal{F}$ be a finite subset of $\mathcal{X}$ and $I_{\mathcal{K}}$ be an ideal of $R[[\mathcal{K}]]$ for each finite subset $\mathcal{K}$

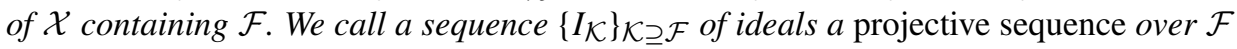
if $\mathcal{K}\left(I_{\mathcal{L}}\right)=I_{\mathcal{K}}$ whenever $\mathcal{F} \subseteq \mathcal{K} \subseteq \mathcal{L}$.

Theorem 3.5. Let $R$ be an integral domain and $J \subseteq I$ be ideals of $R[[\mathcal{X}]]_{3}$, where $I$ is an invertible ideal of $R[[\mathcal{X}]]_{3}$. If $\mathcal{K}(I)=\mathcal{K}(J) \neq(0)$ for some finite subset $\mathcal{K}$ of $\mathcal{X}$, then $\mathcal{K}(I)$ is invertible, and $I=J$. 
Proof. Suppose that $I$ is an invertible ideal of $R[[\mathcal{X}]]_{3}$ containing an ideal $J$ such that $\mathcal{K}(I)=\mathcal{K}(J) \neq(0)$ for some finite subset $\mathcal{K}$ of $\mathcal{X}$. Then $J=I J^{\prime}$, where $J^{\prime}:=I^{-1} J$ is an ideal of $R[[\mathcal{X}]]_{3}$. Since $\mathcal{K}(I)=\mathcal{K}(J)$, it follows from the $\mathcal{K}$-projection of $J$ that

$$
\mathcal{K}(I)=\mathcal{K}(J)=\mathcal{K}(I) \mathcal{K}\left(J^{\prime}\right) .
$$

Since $\mathcal{K}(I)$ is an invertible ideal of $R[[\mathcal{K}]]$ by Lemma 3.1, we cancel $\mathcal{K}(I)$ and obtain the equality $\mathcal{K}\left(J^{\prime}\right)=R[[\mathcal{K}]]$. Therefore we can choose $f \in J^{\prime}$ such that $\mathcal{K}(f)=1$. By Theorem 2.1 (3), $f$ is a unit in $R[[\mathcal{X}]]_{3}$. Thus $J^{\prime}=R[[\mathcal{X}]]_{3}$. Hence $J=I J^{\prime}=I$.

Theorem 3.6. Let $I$ and $J$ be ideals of $R[[\mathcal{X}]]_{3}$, where $R$ is an integral domain, such that $\mathcal{F}(I)=\mathcal{F}(J)$ for every finite subset $\mathcal{F}$ of $\mathcal{X}$. If $I$ is an invertible ideal, then $I=J$.

Proof. Choose $0 \neq a \in I$. Put $H:=\left(a I^{-1}\right) J$, which is an ideal of $R[[\mathcal{X}]]_{3}$. Note that since $0 \neq a \in I$, there exists a finite subset $\mathcal{F}$ of $\mathcal{X}$ such that $\mathcal{K}(a) \neq 0$ for every finite subset $\mathcal{K} \supseteq \mathcal{F}$ of $\mathcal{X}$. Thus $\mathcal{K}(I) \neq 0$ for every finite subset $\mathcal{K} \supseteq \mathcal{F}$ of $\mathcal{X}$. We have $\mathcal{K}(H)=\mathcal{K}\left(a I^{-1}\right) \mathcal{K}(J)$. We show $\mathcal{K}\left(a I^{-1}\right)=\mathcal{K}(a) \mathcal{K}(I)^{-1}$. By taking the $\mathcal{K}$-projection of the equation $(a)=\left(a I^{-1}\right) I$, we get $(\mathcal{K}(a))=\mathcal{K}\left(a I^{-1}\right) \mathcal{K}(I)$. By Lemma 3.1. $\mathcal{K}(I)$ is an invertible ideal of $R[[\mathcal{K}]]$, so $\mathcal{K}\left(a I^{-1}\right)=\mathcal{K}(a) \mathcal{K}(I)^{-1}$. Now

$$
\mathcal{K}(H)=\mathcal{K}(a) \mathcal{K}(I)^{-1} \mathcal{K}(J)=\mathcal{K}(a) \mathcal{K}(I)^{-1} \mathcal{K}(I)=(\mathcal{K}(a))
$$

Thus $\mathcal{K}(H)=(\mathcal{K}(a))$ for every finite subset $\mathcal{K} \supseteq \mathcal{F}$ of $\mathcal{X}$. We show $H \subseteq(a)$. Let $h \in H$. Choose $\gamma_{\mathcal{K}} \in R[[\mathcal{K}]]$ such that $\mathcal{K}(h)=\gamma_{\mathcal{K}} \mathcal{K}(a)$ for every finite subset $\mathcal{K} \supseteq \mathcal{F}$ of $\mathcal{X}$. It is easy to see that $\left\{\gamma_{\mathcal{K}}\right\}_{\mathcal{K} \supseteq \mathcal{F}}$ is a projective sequence. By Theorem 2.1(5), there exists $\gamma \in R[[\mathcal{X}]]_{3}$ such that $\mathcal{K}(\gamma)=\gamma_{\mathcal{K}}$ for every finite subset $\mathcal{K} \supseteq \mathcal{F}$ of $\mathcal{X}$. Now $\mathcal{K}(h)=\mathcal{K}(\gamma) \mathcal{K}(a)=\mathcal{K}(\gamma a)$. By Theorem 2.1 (4), $h=\gamma a$ and hence $H \subseteq(a)$. Applying Theorem 3.5, we deduce $H=(a)$, whence $a I^{-1} J=(a)$. Cancelling $(a)$ from this equation, we get the equality $I^{-1} J=R[[\mathcal{X}]]_{3}$, and hence $I=J$.

We present an example which justifies the condition that " $I$ is an invertible ideal" in Theorems 3.5 and 3.6

Example 3.7. Let $R$ be an integral domain and $\mathcal{X}$ be an infinite set of indeterminates. Put

$$
\left\{\begin{array}{l}
(\mathcal{X})_{2}:=\left\{f \mid f \in R[[\mathcal{X}]]_{2}, f(0)=0\right\}, \\
(\mathcal{X})_{3}:=\left\{f \mid f \in R[[\mathcal{X}]]_{3}, f(0)=0\right\} .
\end{array}\right.
$$

Put $J:=(\mathcal{X})_{2} R[[\mathcal{X}]]_{3}$ and $I:=(\mathcal{X})_{3}$. It is clear that $J \subseteq I$. Let $f \in I$. Then for every finite subset $\mathcal{K}$ of $\mathcal{X}$, we have $\mathcal{K}(f) \in(\mathcal{X})_{2}$ and $\mathcal{K}(f)=\mathcal{K}(\mathcal{K}(f))$, hence $\mathcal{K}(f) \in \mathcal{K}(J)$. So $\mathcal{K}(I) \subseteq \mathcal{K}(J)$. Thus $\mathcal{K}(I)=\mathcal{K}(J)$ for every finite subset $\mathcal{K}$ of $\mathcal{X}$. We claim that $I \neq J$. Put $f:=X_{1}+X_{2}+\cdots=\sum_{i=1}^{\infty} X_{i}$. Thus $f \in I$. We claim $f \notin J$. Suppose that $f \in J=(\mathcal{X})_{2} R[[\mathcal{X}]]_{3}$. Then

$$
f=X_{1}+X_{2}+\cdots=f_{1} g_{1}+\cdots+f_{n} g_{n},
$$


where $f_{i} \in(\mathcal{X})_{2}$ and $g_{i} \in R[[\mathcal{X}]]_{3}$ for $i=1, \ldots, n$. Since $f_{i}(0)=0$ for each $i$, comparing the first degree terms, we have

$$
f=X_{1}+X_{2}+\cdots=f_{11} g_{10}+\cdots+f_{n 1} g_{n 0},
$$

where $f_{i 1}$ is the first degree form of $f_{i}$ and $g_{i 0}=g_{i}(0)$ for each $i$. But since $f_{i} \in(\mathcal{X})_{2}$, $f_{i 0}$ has only finitely many monomials of degree 1 . Thus $f=X_{1}+X_{2}+\cdots$ has only finitely many monomials of degree 1 , which is a contradiction.

Next we study when the condition " $\mathcal{F}(J) \subseteq \mathcal{F}(I)$ for all $\mathcal{F}$ " forces $J \subseteq I$.

Theorem 3.8. Let $I$ and $J$ be ideals of $R[[\mathcal{X}]]_{3}$, where $R$ is an integral domain, such that $\mathcal{F}(J) \subseteq \mathcal{F}(I)$ for every finite subset $\mathcal{F}$ of $\mathcal{X}$. If I is an invertible ideal, then $J \subseteq I$.

Proof. Let $0 \neq a \in J$. Then there exists a finite subset $\mathcal{F}$ such that $\mathcal{K}(a) \neq 0$ for every finite subset $\mathcal{K} \supseteq \mathcal{F}$ of $\mathcal{X}$. Since $0 \neq \mathcal{K}(a) \in \mathcal{K}(J) \subseteq \mathcal{K}(I)$, by Lemma 3.1, $\mathcal{K}(I)$ is an invertible ideal of $R[[K]]$ for every finite subset $\mathcal{K} \supseteq \mathcal{F}$ of $\mathcal{X}$. Choose $0 \neq b \in I$. Put $H:=a b I^{-1}$, which is an ideal of $R[[\mathcal{X}]]_{3}$. By taking the $\mathcal{K}$-projection of the equation (b) $=\left(b I^{-1}\right) I$, we get $(\mathcal{K}(b))=\mathcal{K}\left(b I^{-1}\right) \mathcal{K}(I)$. Since $\mathcal{K}(I)$ is an invertible ideal of $R\left[[\mathcal{K}]\right.$ ], we have $\mathcal{K}\left(b I^{-1}\right)=\mathcal{K}(b) \mathcal{K}(I)^{-1}$. By applying the $\mathcal{K}$-projection of $H$, since $\mathcal{K}(a) \in \mathcal{K}(I)$, we have

$$
\mathcal{K}(H)=\mathcal{K}(a) \mathcal{K}\left(b I^{-1}\right)=\mathcal{K}(a) \mathcal{K}(b) \mathcal{K}(I)^{-1} \subseteq(\mathcal{K}(b)) .
$$

Thus $\mathcal{K}(H) \subseteq(\mathcal{K}(b))$ for every finite subset $\mathcal{K} \supseteq \mathcal{F}$ of $\mathcal{X}$. We show $H \subseteq(b)$. Let $h \in H$. Choose $\gamma_{\mathcal{K}} \in R[[\mathcal{K}]]$ such that $\mathcal{K}(h)=\gamma_{\mathcal{K}} \mathcal{K}(b)$ for every finite subset $\mathcal{K} \supseteq \mathcal{F}$ of $\mathcal{X}$. It is easy to see that $\left\{\gamma_{\mathcal{K}}\right\}_{\mathcal{K} \supseteq \mathcal{F}}$ is a projective sequence. By Theorem 2.1 (5), there exists $\gamma \in R[[\mathcal{X}]]_{3}$ such that $\mathcal{K}(\gamma)=\gamma_{\mathcal{K}}$ for every finite subset $\mathcal{K} \supseteq \mathcal{F}$ of $\mathcal{X}$. Now $\mathcal{K}(h)=\mathcal{K}(\gamma) \mathcal{K}(b)=\mathcal{K}(\gamma b)$. By Theorem 2.1.4), $h=\gamma b$ and hence $H=a b I^{-1} \subseteq(b)$. Thus $a \in I$. Therefore $J \subseteq I$.

Cashwell and Everett [5] showed that a projective sequence of principal ideals has a primitive ideal in the sense that the projective sequence is the projection of an ideal in $R[[\mathcal{X}]]_{3}$. We show that a projective sequence of invertible ideals also has a primitive ideal in $R[[\mathcal{X}]]_{3}$.

Theorem 3.9. Let $R$ be an integral domain with quotient field $K$, and $\mathcal{X}$ be a set of indeterminates over $R$. If $\left\{I_{\mathcal{F}}\right\}$ is a projective sequence of invertible ideals of $R[[\mathcal{F}]]$ over $\emptyset$, then there exists a unique invertible ideal I of the form $f I_{0}[[\mathcal{X}]]_{3}$ of $R[[\mathcal{X}]]_{3}$, where $f \in K[[\mathcal{X}]]_{3}$ and $I_{0}:=I_{\emptyset}$ is an invertible ideal of $R$, such that $\mathcal{F}(I)=I_{\mathcal{F}}$ in $R[[\mathcal{F}]]$ for every finite subset $\mathcal{F}$ of $\mathcal{X}$.

Proof. Let $\left\{I_{\mathcal{F}}\right\}_{\mathcal{F} \supseteq \emptyset}$ be a projective sequence of invertible ideals of $R[[\mathcal{F}]]$ over $\emptyset$. Put $I_{0}:=I_{\emptyset}$. Since $I_{\mathcal{F}}$ is an invertible ideal of $R[[\mathcal{F}]]$ for each finite subset $\mathcal{F}$ of $\mathcal{X}$, it follows from Corollary 3.3 that

$$
I_{\mathcal{F}}=f_{\mathcal{F}} I_{0}[[\mathcal{F}]], \quad \text { where } \quad f_{\mathcal{F}} \in K[[\mathcal{F}]] .
$$

Since $I_{0} \neq(0)$, choose $0 \neq b \in I_{0}$ so that $b f_{\mathcal{F}} \in R[[\mathcal{F}]]$ for every $\mathcal{F}$. 
We claim that $\left\{b f_{\mathcal{F}}\right\}_{\mathcal{F} \supseteq \emptyset}$ is an associative sequence. For any pair of finite subsets $\mathcal{F}$ and $\mathcal{K}$ of $\mathcal{X}$ such that $\mathcal{F} \subseteq \mathcal{K}$, we have $b I_{\mathcal{K}}=b f_{\mathcal{K}} I_{0}[[\mathcal{K}]]$ and $b I_{\mathcal{F}}=b f_{\mathcal{F}} I_{0}[[\mathcal{F}]]$. Since $b \in I_{0}$ and $\left\{I_{\mathcal{F}}\right\}_{\mathcal{F} \supseteq \emptyset}$ is projective, it follows from the $\mathcal{F}$-projection of $b I_{\mathcal{K}}$ that

$$
b f_{\mathcal{F}} I_{0}[[\mathcal{F}]]=b I_{\mathcal{F}}=\mathcal{F}\left(b I_{\mathcal{K}}\right)=\mathcal{F}\left(b f_{\mathcal{K}} I_{0}[[\mathcal{K}]]\right)=\mathcal{F}\left(b f_{\mathcal{K}}\right) I_{0}[[\mathcal{F}]]
$$

Since $I_{0}$ is an invertible ideal of $R, I_{0}[[\mathcal{F}]]$ is also an invertible ideal of $R[[\mathcal{F}]]$. We cancel $I_{0}[[\mathcal{F}]]$ and obtain the equality $b f_{\mathcal{F}} R[[\mathcal{F}]]=\mathcal{F}\left(b f_{\mathcal{K}}\right) R[[\mathcal{F}]]$. Therefore, $b f_{\mathcal{F}} \sim \mathcal{F}\left(b f_{\mathcal{K}}\right)$ for any pair of finite subsets $\mathcal{F}$ and $\mathcal{K}$ of $\mathcal{X}$ such that $\mathcal{F} \subseteq \mathcal{K}$. Thus the claim is proved.

Since $\left\{b f_{\mathcal{F}}\right\}_{\mathcal{F} \supseteq \emptyset}$ is an associative sequence, by Theorem 2.1 (6) there exists an element $f \in R[[\mathcal{X}]]_{3}$ such that $\mathcal{F}(f) \sim b f_{\mathcal{F}}$ for every finite subset $\mathcal{F}$ of $\mathcal{X}$. Thus $\mathcal{F}(f) R[[\mathcal{F}]]=$ $b f_{\mathcal{F}} R[[\mathcal{F}]]$. Put $I=b^{-1} f I_{0}[[\mathcal{X}]]_{3}$. Since $\mathcal{F}(f) \sim b f_{\mathcal{F}}$ for every finite subset $\mathcal{F}$ of $\mathcal{X}$, we have $\mathcal{F}(I)=b^{-1} \mathcal{F}(f) I_{0}[[\mathcal{F}]]=f_{\mathcal{F}} I_{0}[[\mathcal{F}]]=I_{\mathcal{F}}$. Thus $\mathcal{F}(I)=I_{\mathcal{F}}$ for every finite subset $\mathcal{F}$ of $\mathcal{X}$. So $I \subseteq R[[\mathcal{X}]]_{3}$. It is clear that $I=b^{-1} f I_{0}[[\mathcal{X}]]_{3}$ is an invertible ideal of $R[[\mathcal{X}]]_{3}$. The uniqueness of $I$ follows from Theorem 3.6

Corollary 3.10. Let $R$ be an integral domain with quotient field $K$, and $\mathcal{X}$ be a set of

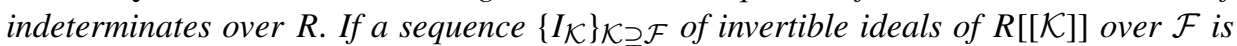
projective, then there exists a unique invertible ideal I of the form $f I^{\prime}[[\mathcal{X}]]_{3}$ in $R[[\mathcal{X}]]_{3}$, where $f \in K[[\mathcal{X}]]_{3}$ and $I^{\prime}$ is an invertible ideal of $R$, such that $\mathcal{K}(I)=I_{\mathcal{K}}$ in $R[[\mathcal{F}]]$ for every finite subset $\mathcal{K} \supseteq \mathcal{F}$ of $\mathcal{X}$.

Proof. Put $D:=R[[\mathcal{F}]]$. Note that $R[[\mathcal{X}]]_{3}=D\left[\left[\mathcal{X}^{\prime}\right]\right]_{3}$, where $\mathcal{X}^{\prime}=\mathcal{X} \backslash \mathcal{F}$. Then $\left\{I_{\mathcal{K}}\right\}_{\mathcal{K} \supseteq \mathcal{F}}=\left\{I_{\mathcal{K}^{\prime}}\right\}_{\mathcal{K}^{\prime} \supseteq \emptyset}$ is a projective sequence of invertible ideals of $D\left[\left[\mathcal{K}^{\prime}\right]\right]$. By Theorem 3.9 there exists an invertible ideal $I:=g I_{\mathcal{F}}\left[\left[\mathcal{X}^{\prime}\right]\right]_{3}$ of $D\left[\left[\mathcal{X}^{\prime}\right]\right]_{3}=R[[\mathcal{X}]]_{3}$ such that $\mathcal{K}(I)=I_{\mathcal{K}}$ for every finite subset $\mathcal{K} \supseteq \mathcal{F}$ of $\mathcal{X}$. Since $I_{\mathcal{F}}$ is an invertible ideal of $D=R[[\mathcal{F}]]$, by applying Corollary 3.3 to $I_{\mathcal{F}}$, we have

$$
I=g\left(h I^{\prime}[[\mathcal{F}]]\right)[[\mathcal{X} \backslash \mathcal{F}]]_{3}=f I^{\prime}[[\mathcal{X}]]_{3},
$$

where $f=g h$ and $I^{\prime}$ is an invertible ideal of $R$. Note that $f \in K[[\mathcal{X}]]_{3}$ since af $\in I \subseteq$ $R[[\mathcal{X}]]_{3}$, where $0 \neq a \in I^{\prime}$.

We establish a relation between invertible ideals of $R[[\mathcal{X}]]_{3}$ and their projections.

Theorem 3.11. Let $R$ be an integral domain with quotient field $K$. If $I$ is an invertible ideal of $R[[\mathcal{X}]]_{3}$, then $I=f I^{\prime}[[\mathcal{X}]]_{3}$, where $I^{\prime}$ is an invertible ideal of $R$ and $f \in$ $K[[\mathcal{X}]]_{3}$. Moreover, if $I_{0}=\emptyset(I) \neq(0)$, then $I=f I_{0}[[\mathcal{X}]]_{3}$, where $f \in K[[\mathcal{X}]]_{3}$.

Proof. Let $I$ be an invertible ideal of $R[[\mathcal{X}]]_{3}$. Put $I_{0}=\emptyset(I)$. We will divide the proof into two cases; either $I_{0} \neq(0)$ or $I_{0}=(0)$.

Case $\mathrm{I}: I_{0} \neq(0)$ (note that $I_{0}$ is an invertible ideal of $R$ by Lemma 3.1).

By Lemma 3.1, $\mathcal{K}(I)$ is an invertible ideal of $R[[\mathcal{K}]]$ for every finite subset $\mathcal{K}$ of $\mathcal{X}$. Since $\{\mathcal{K}(I)\}_{\mathcal{K} \supseteq \emptyset}$ is a projective sequence of invertible ideals, by Theorem 3.9 there exists an invertible ideal $I^{\prime}=f I_{0}[[\mathcal{X}]]_{3}$ of $R[[\mathcal{X}]]_{3}$, where $f \in K[[\mathcal{X}]]_{3}$ such that $\mathcal{K}\left(I^{\prime}\right)=$ $\mathcal{K}(f) I_{0}[[\mathcal{K}]]=\mathcal{K}(I)$ for every finite subset $\mathcal{K}$ of $\mathcal{X}$. We claim $I \subseteq I^{\prime}=f I_{0}[[\mathcal{X}]]_{3}$. Let $i \in I$. Since $\mathcal{K}(I)=\mathcal{K}(f) I_{0}[[\mathcal{K}]]$, we have $\mathcal{K}(i)=\mathcal{K}(f) a_{\mathcal{K}}$, where $a_{\mathcal{K}} \in I_{0}[[\mathcal{K}]]$. Note 


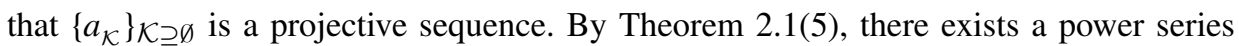
$a \in R[[\mathcal{X}]]_{3}$ such that $\mathcal{K}(a)=a_{\mathcal{K}}$ for every finite subset $\mathcal{K}$ of $\mathcal{X}$. Clearly $a \in I_{0}[[\mathcal{X}]]_{3}$ since $\mathcal{K}(a)=a_{\mathcal{K}} \in I_{0}[[\mathcal{K}]]$ for every finite subset $\mathcal{K}$ of $\mathcal{X}$. Therefore, for every finite subset $\mathcal{K}$ of $\mathcal{X}$, we have

$$
\mathcal{K}(i)=\mathcal{K}(f) a_{\mathcal{K}}=\mathcal{K}(f) \mathcal{K}(a)=\mathcal{K}(f a)
$$

By Theorem 2.1 $(4), i=f a \in f I_{0}[[\mathcal{X}]]_{3}$. Thus the claim is proved. Since $I^{\prime}=f I_{0}[[\mathcal{X}]]_{3}$ is an invertible ideal of $R[[\mathcal{X}]]_{3}$ containing $I$, and $\mathcal{K}(I)=\mathcal{K}\left(I^{\prime}\right)$, we can apply Theorem 3.5 and obtain the equality $I=I^{\prime}=f I_{0}[[\mathcal{X}]]_{3}$.

Case II : $\mathcal{K}(I) \neq(0)$ for some finite subset $\mathcal{K}$ of $\mathcal{X}$ (note that $\mathcal{K}(I)$ is an invertible ideal of $R[[\mathcal{K}]]$ by Lemma 3.1].

Let $D=R[[\mathcal{K}]]$. Then $R[[\mathcal{X}]]_{3}=D\left[\left[\mathcal{X}^{\prime}\right]\right]_{3}$, where $\mathcal{X}^{\prime}=\mathcal{X} \backslash \mathcal{K}$. By Case I, $I=g J\left[\left[\mathcal{X}^{\prime}\right]\right]_{3}$, where $J=\mathcal{K}(I)$ is an invertible ideal of $R[[\mathcal{K}]]$. By Corollary 3.3 $J=h I^{\prime}[[\mathcal{K}]]$, where $I^{\prime}$ is an invertible ideal of $R$. Therefore, $I=g h I^{\prime}\left[\left[\mathcal{K} \cup \mathcal{X}^{\prime}\right]\right]_{3}=$ $f I^{\prime}[[\mathcal{X}]]_{3}$, where $f=g h$. Note that $f \in K[[\mathcal{X}]]_{3}$ since af $\in I \subseteq R[[\mathcal{X}]]_{3}$, where $0 \neq a \in I^{\prime}$.

Let $R$ be an integral domain. The set $\mathcal{I}(R)$ of invertible fractional ideals of $R$ is a group under ideal multiplication. The set $\mathcal{P}(R)$ of principal fractional ideals of $R$ forms a subgroup of $\mathcal{I}(R)$. The factor group $\mathcal{I}(R) / \mathcal{P}(R)$ is called the Picard group of $R$. In the case when $R$ is a Krull domain, let $\mathcal{D}(R)$ be the group of divisorial ideals of $R$. Then $\mathcal{D}(R) / \mathcal{P}(R)$ is called the divisor class group of $R$. We denote the divisor class group and Picard group of $R$ by $\mathrm{Cl}(R)$ and $\operatorname{Pic}(R)$, respectively.

It is well known that $\operatorname{Pic}(R) \cong \operatorname{Pic}(R[[X]])$ in the single variable case. This result can be generalized to an arbitrary number of variables using Theorem 3.11 .

Corollary 3.12. Let $R$ be an integral domain. The natural mapping $J \mapsto J[[\mathcal{X}]]_{3}$, where $J$ is an ideal of $R$, induces an isomorphism $\operatorname{Pic}(R) \cong \operatorname{Pic}\left(R[[\mathcal{X}]]_{3}\right)$.

\section{4. $R[[\mathcal{X}]]_{3}$ is a $\pi$-domain if $R$ is a formally stable $\pi$-domain}

Let $R$ be an integral domain and $\mathcal{X}$ be a set of indeterminates over $R$. If $R\left[\left[X_{1}, \ldots, X_{n}\right]\right]$ is a $\pi$-domain for each finite set $\left\{X_{1}, \ldots, X_{n}\right\}$ of indeterminates over $R$, then we say that $R$ is a formally stable $\pi$-domain. Note that if $R$ is a formally stable $\pi$-domain, then $R$ is also a $\pi$-domain. In this section, we will prove that if $R$ is a formally stable $\pi$-domain, then $R[[\mathcal{X}]]_{3}$ is also a $\pi$-domain.

If $R$ is a $\pi$-domain, then every principal ideal $(a)$ of $R$ is a product of a finite number of prime ideals of $R$. Since the prime ideals in this factorization are invertible, the factorization is unique. We denote by $\|a\|$ and $N(a)$ the number of prime ideals and distinct prime ideals in the ideal factorization of $(a)$, respectively.

Lemma 4.1. Let $\delta$ be a nonunit in $R[[\mathcal{X}]]_{3}$, where $R$ is a formally stable $\pi$-domain. Then

(i) $\|\mathcal{K}(\delta)\| \leq\|\mathcal{F}(\delta)\|$ whenever $\mathcal{F} \subseteq \mathcal{K}$, where $\mathcal{F}$ and $\mathcal{K}$ are finite subsets of $\mathcal{X}$, 
(ii) there exists a finite subset $\mathcal{F}_{\delta}$, depending on $\delta$, of $\mathcal{X}$ such that for every finite subset $\mathcal{K} \supseteq \mathcal{F}_{\delta}$ of $\mathcal{X},\|\mathcal{K}(\delta)\|=\left\|\mathcal{F}_{\delta}(\delta)\right\|$; furthermore, if $\left(\mathcal{F}_{\delta}(\delta)\right)=P_{1, \mathcal{F}_{\delta}} \cdots P_{n, \mathcal{F}_{\delta}}$ and $(\mathcal{K}(\delta))=P_{1, \mathcal{K}} \cdots P_{n, \mathcal{K}}$, where $P_{i, \mathcal{F}}$ 's and $P_{i, \mathcal{K}}$ 's are prime ideals of $R[[\mathcal{F}]]$ and $R[[\mathcal{K}]]$, respectively, then $\mathcal{F}_{\delta}\left(P_{i, \mathcal{K}}\right)=P_{i, \mathcal{F}_{\delta}}$ for $i=1, \ldots, n$ after rearranging the indices.

Proof. (i) Since $\delta$ is a nonunit, it follows from Theorem 2.1 that $\mathcal{F}(\delta)$ is a nonunit in $R[[\mathcal{F}]]$ for any finite subset $\mathcal{F}$ of $\mathcal{X}$. Thus $\|\mathcal{F}(\delta)\| \geq 0$. Let $\mathcal{F} \subseteq \mathcal{K}$ be any pair of finite subsets of $\mathcal{X}$ such that $\|\mathcal{F}(\delta)\|=n$, and $\|\mathcal{K}(\delta)\|=m$. Suppose that $n<m$. Since $R$ is a formally stable $\pi$-domain, $(\mathcal{F}(\delta))=P_{1, \mathcal{F}} \cdots P_{n, \mathcal{F}}$ and $(\mathcal{K}(\delta))=P_{1, \mathcal{K}} \cdots P_{m, \mathcal{K}}$, where $P_{i, \mathcal{F}}$ 's and $P_{j, \mathcal{K}}$ 's are (not necessarily distinct) prime ideals of $R[[\mathcal{F}]]$ and $R[[\mathcal{K}]]$, respectively. From Theorem 2.1, we obtain

$$
\mathcal{F}(\mathcal{K}(\delta))=\mathcal{F}\left(P_{1, \mathcal{K}} \cdots P_{m, \mathcal{K}}\right)=\mathcal{F}\left(P_{1, \mathcal{K}}\right) \cdots \mathcal{F}\left(P_{m, \mathcal{K}}\right)=P_{1, \mathcal{F}} \cdots P_{n, \mathcal{F}}=(\mathcal{F}(\delta)) .
$$

Since $P_{1, \mathcal{F}}$ is a prime ideal of $R[[\mathcal{F}]]$, we may assume that $\mathcal{F}\left(P_{1, \mathcal{K}}\right) \subseteq P_{1, \mathcal{F}}$. Moreover, it follows from the invertibility of $P_{1, \mathcal{F}}$ that $\mathcal{F}\left(P_{1, \mathcal{K}}\right)=P_{1, \mathcal{F}} I_{1, \mathcal{F}}$ for some ideal $I_{1, \mathcal{F}}$ of $R[[\mathcal{F}]]$. Note that $I_{1, \mathcal{F}}$ need not be a proper ideal. By cancellation, we get

$$
I_{1, \mathcal{F}} \mathcal{F}\left(P_{2, \mathcal{K}}\right) \cdots \mathcal{F}\left(P_{m, \mathcal{K}}\right)=P_{2, \mathcal{F}} P_{3, \mathcal{F}} \cdots P_{n, \mathcal{F}}
$$

Since $n<m$, if we continue this process on $P_{i, \mathcal{F}}$ 's for $i=2, \ldots, n$, then there exists at least one $P_{j, \mathcal{K}}$ such that $R[[\mathcal{F}]]=\mathcal{F}\left(P_{j, \mathcal{K}}\right) I$, where $I$ is an ideal of $R[[\mathcal{F}]]$. Since $P_{j, \mathcal{K}}$ is a proper ideal of $R[[\mathcal{K}]], \mathcal{F}\left(P_{j, \mathcal{K}}\right)$ is a proper ideal of $R[[\mathcal{F}]]$. This is a contradiction. Therefore, $\|\mathcal{K}(\delta)\| \leq\|\mathcal{F}(\delta)\|$.

(ii) Suppose that there are no finite subsets of $\mathcal{X}$ satisfying the condition above. Then there exists an infinite chain $\left\{\mathcal{F}_{i}\right\}$ of finite subsets of $\mathcal{X}$ such that $\mathcal{F}_{1} \subseteq \mathcal{F}_{2} \subseteq \cdots$ and $\left\|\mathcal{F}_{1}(\delta)\right\|>\left\|\mathcal{F}_{2}(\delta)\right\|>\cdots$, which contradicts the finiteness of $\left\|\mathcal{F}_{1}(\delta)\right\|$. Hence there exists a finite subset $\mathcal{F}_{\delta}$, depending on $\delta$, of $\mathcal{X}$ such that for every finite subset $\mathcal{K} \supseteq \mathcal{F}_{\delta}$ of $\mathcal{X},\|\mathcal{K}(\delta)\|=\left\|\mathcal{F}_{\delta}(\delta)\right\|$.

Let $\left(\mathcal{F}_{\delta}(\delta)\right)=P_{1, \mathcal{F}_{\delta}} \cdots P_{n, \mathcal{F}_{\delta}}$ and $(\mathcal{K}(\delta))=P_{1, \mathcal{K}} \cdots P_{n, \mathcal{K}}$. Since $\|\mathcal{K}(\delta)\|=\left\|\mathcal{F}_{\delta}(\delta)\right\|$, by the same argument as in the proof of (i), we may assume that $\mathcal{F}_{\delta}\left(P_{i, \mathcal{K}}\right) \subseteq P_{i, \mathcal{F}_{\delta}}$ and $\mathcal{F}_{\delta}\left(P_{i, \mathcal{K}}\right)=P_{i, \mathcal{F}_{\delta}} I_{i, \mathcal{F}_{\delta}}$ for $i=1, \ldots, n$, after rearranging the indices. By cancellation, we can get

$$
I_{1, \mathcal{F}_{\delta}} \cdots I_{n, \mathcal{F}_{\delta}}=R\left[\left[\mathcal{F}_{\delta}\right]\right]
$$

Since $I_{i, \mathcal{F}_{\delta}}$ is an ideal of $R\left[\left[\mathcal{F}_{\delta}\right]\right], I_{i, \mathcal{F}_{\delta}}=R\left[\left[\mathcal{F}_{\delta}\right]\right]$ and $\mathcal{F}_{\delta}\left(P_{i, \mathcal{K}}\right)=P_{i, \mathcal{F}_{\delta}}$ for $i=$ $1, \ldots, n$.

Remark. (1) For any finite subset $\mathcal{K}$ of $\mathcal{X}$ containing $\mathcal{F}_{\delta}$, if $P_{\mathcal{K}}$ is a prime ideal of $R[[\mathcal{K}]]$ appearing in the ideal factorization of $\mathcal{K}(\delta)$, then $\mathcal{F}_{\delta}\left(P_{\mathcal{K}}\right)$ is a prime ideal of $R\left[\left[\mathcal{F}_{\delta}\right]\right]$ appearing in the ideal factorization of $\mathcal{F}_{\delta}(\delta)$.

(2) Although $P_{\mathcal{K}}$ and $P_{\mathcal{K}}^{\prime}$ are prime ideals of $R[[\mathcal{K}]]$ appearing in the ideal factorization of $\mathcal{K}(\delta)$ such that $\mathcal{F}_{\delta}\left(P_{\mathcal{K}}\right)=\mathcal{F}_{\delta}\left(P_{\mathcal{K}}^{\prime}\right), P_{\mathcal{K}}$ and $P_{\mathcal{K}}^{\prime}$ need not be the same. Thus it may happen that $N(\mathcal{K}(\delta)) \neq N\left(\mathcal{F}_{\delta}(\delta)\right)$ even if $\|\mathcal{K}(\delta)\|=\left\|\mathcal{F}_{\delta}(\delta)\right\|$.

Lemma 4.2. Let $\delta$ be a nonunit in $R[[\mathcal{X}]]_{3}$, where $R$ is a formally stable $\pi$-domain. Then

(i) $N\left(\mathcal{F}_{\delta}(\delta)\right) \leq N(\mathcal{K}(\delta))$ for any finite subset $\mathcal{K}$ of $\mathcal{X}$ containing $\mathcal{F}_{\delta}$, 
(ii) there exists a finite subset $\mathcal{F}_{0}$ of $\mathcal{X}$ such that for every finite subset $\mathcal{K}$ of $\mathcal{X}$ containing $\mathcal{F}_{0}, N\left(\mathcal{F}_{0}(\delta)\right)=N(\mathcal{K}(\delta))$ and $\left\|\mathcal{F}_{0}(\delta)\right\|=\|\mathcal{K}(\delta)\|$.

Proof. (i) Let $P_{\mathcal{K}}$ be a prime ideal of $R[[\mathcal{K}]]$ in the ideal factorization of $\mathcal{K}(\delta)$. Since $\mathcal{F}_{\delta}\left(P_{\mathcal{K}}\right)$ is a prime ideal of $R[[\mathcal{F}]]$ containing $\mathcal{F}_{\delta}(\delta)$, it is exactly one of prime ideals of $R[[\mathcal{F}]]$ in the ideal factorization of $\mathcal{F}_{\delta}(\delta)$. Therefore, $N\left(\mathcal{F}_{\delta}(\delta)\right) \leq N(\mathcal{K}(\delta))$.

(ii) For any pair of finite subsets $\mathcal{K}$ and $\mathcal{L}$ of $\mathcal{X}$ containing $\mathcal{F}_{\delta}$ such that $\mathcal{K} \subseteq \mathcal{L}$, we have $N\left(\mathcal{F}_{\delta}(\delta)\right) \leq N(\mathcal{K}(\delta)) \leq N(\mathcal{L}(\delta))$ and $\left\|\mathcal{F}_{\delta}(\delta)\right\|=\|\mathcal{K}(\delta)\|=\|\mathcal{L}(\delta)\|$. Since $N(\mathcal{K}(\delta)) \leq\|\mathcal{K}(\delta)\|=\left\|\mathcal{F}_{\delta}(\delta)\right\|$, and $\left\|\mathcal{F}_{\delta}(\delta)\right\|$ is finite, we can choose a finite subset $\mathcal{F}_{0}$ of $\mathcal{X}$ containing $\mathcal{F}_{\delta}$ such that for any finite subset $\mathcal{K} \supseteq \mathcal{F}_{0}$ of $\mathcal{X}, N\left(\mathcal{F}_{0}(\delta)\right)=N(\mathcal{K}(\delta))$ and $\left\|\mathcal{F}_{0}(\delta)\right\|=\|\mathcal{K}(\delta)\|$.

Suppose that $\left(\mathcal{F}_{0}(\delta)\right)=P_{1, \mathcal{F}_{0}}^{m_{1}} \cdots P_{n, \mathcal{F}_{0}}^{m_{n}}$, where $P_{i, \mathcal{F}_{0}}$ 's are distinct prime ideals of $R\left[\left[\mathcal{F}_{0}\right]\right]$. Since $N\left(\mathcal{F}_{0}(\delta)\right)=N(\mathcal{K}(\delta))$ and $\left\|\mathcal{F}_{0}(\delta)\right\|=\|\mathcal{K}(\delta)\|$ for every finite subset $\mathcal{K} \supseteq \mathcal{F}_{0}$ of $\mathcal{X}$, we may assume that $(\mathcal{K}(\delta))=P_{1, \mathcal{K}}^{m_{1}} \cdots P_{n, \mathcal{K}}^{m_{n}}$ and $\mathcal{F}_{0}\left(P_{i, \mathcal{K}}\right)=P_{i, \mathcal{F}_{0}}$ for each $i$, by rearranging the indices.

The following is an immediate consequence of the observation above.

Proposition 4.3. Let $\delta$ be a nonunit in $R[[\mathcal{X}]]_{3}$, where $R$ is a formally stable $\pi$-domain. Suppose that $\left(\mathcal{F}_{0}(\delta)\right)=P_{1, \mathcal{F}_{0}}^{m_{1}} \cdots P_{n, \mathcal{F}_{0}}^{m_{n}}$ is the factorization of $\mathcal{F}_{0}(\delta)$, where $\mathcal{F}_{0}$ is a finite subset of $\mathcal{X}$ defined in Lemma 4.2. Then, for every finite subset $\mathcal{K}$ of $\mathcal{X}$ containing $\mathcal{F}_{0}$, $(\mathcal{K}(\delta))=P_{1, \mathcal{K}}^{m_{1}} \cdots P_{n, \mathcal{K}}^{m_{n}}$, and $\left\{P_{i, \mathcal{K}}\right\}_{\mathcal{K} \supseteq \mathcal{F}}$ is a projective sequence for $i=1, \ldots, n$.

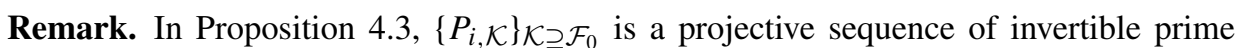
ideals of $R[[\mathcal{K}]]$. According to Corollary 3.10 , there exists an invertible ideal $Q_{i}$ of the form $f P_{i}^{\prime}[[\mathcal{X}]]_{3}$ in $R[[\mathcal{X}]]_{3}$, where $f \in K[[\mathcal{X}]]_{3}$ and $P_{i}^{\prime}$ is an invertible ideal of $R$, such that $\mathcal{K}\left(Q_{i}\right)=P_{i, \mathcal{K}}$ for every finite subset $\mathcal{K} \supseteq \mathcal{F}_{0}$ of $\mathcal{X}$.

From a projective sequence of prime ideals, we will produce a prime ideal.

Lemma 4.4. Let $R$ be an integral domain and $\mathcal{F}$ be a finite subset of $\mathcal{X}$. Suppose that $\left\{P_{\mathcal{K}}\right\}_{\mathcal{K} \supseteq \mathcal{F}}$ is a projective sequence of prime ideals, where $\mathcal{K}$ is an arbitrary finite subset of $\mathcal{X}$ containing $\mathcal{F}$. Then the set $\left\{\alpha \in R[[\mathcal{X}]]_{3} \mid \mathcal{K}(\alpha) \in P_{\mathcal{K}}\right.$ for every finite $\left.\mathcal{K} \supseteq \mathcal{F}\right\}$ is a prime ideal of $R[[\mathcal{X}]]_{3}$.

Proof. Put $P_{\infty}=\left\{\alpha \in R[[\mathcal{X}]]_{3} \mid \mathcal{K}(\alpha) \in P_{\mathcal{K}}\right.$ for every finite $\left.\mathcal{K} \supseteq \mathcal{F}\right\}$. Since $0 \in P_{\infty}$, $P_{\infty}$ is a nonempty set. Since the map $\alpha \mapsto \mathcal{K}(\alpha)$ is a ring homomorphism, $P_{\infty}$ is an ideal of $R[[\mathcal{X}]]_{3}$. Suppose that $\alpha \notin P_{\infty}$ and $\beta \notin P_{\infty}$. Since $\left\{P_{\mathcal{K}}\right\}_{\mathcal{K} \supseteq \mathcal{F}}$ is a projective sequence, we can choose finite subsets $\mathcal{F}_{\alpha}$ and $\mathcal{F}_{\beta}$ of $\mathcal{X}$ containing $\mathcal{F}$ such that $\mathcal{F}_{\alpha}(\alpha) \notin P_{\mathcal{F}_{\alpha}}$ and $\mathcal{F}_{\beta}(\beta) \notin P_{\mathcal{F}_{\beta}}$. Note that $\mathcal{K}(\alpha) \notin P_{\mathcal{K}}$ and $\mathcal{L}(\beta) \notin P_{\mathcal{L}}$ for any finite subsets $\mathcal{K} \supseteq \mathcal{F}_{\alpha}$ and $\mathcal{L} \supseteq \mathcal{F}_{\beta}$. Put $\mathcal{F}^{\prime}=\mathcal{F}_{\alpha} \cup \mathcal{F}_{\beta}$. Note that $\mathcal{F} \subseteq \mathcal{F}^{\prime}, \mathcal{K}(\alpha) \notin P_{\mathcal{K}}$, and $\mathcal{K}(\beta) \notin P_{\mathcal{K}}$ for every finite subset $\mathcal{K} \supseteq \mathcal{F}^{\prime}$ of $\mathcal{X}$. Since $P_{\mathcal{K}}$ is a prime ideal, $\mathcal{K}(\alpha \beta)=\mathcal{K}(\alpha) \mathcal{K}(\beta) \notin P_{\mathcal{K}}$. Thus $\alpha \beta \notin P_{\infty}$. Therefore $P_{\infty}$ is a prime ideal of $R[[\mathcal{X}]]_{3}$.

Now we are ready to show that $R[[\mathcal{X}]]_{3}$ is a $\pi$-domain over a formally stable $\pi$-domain $R$. 
Theorem 4.5. Let $R$ be an integral domain and $\mathcal{X}$ be a set of indeterminates. If $R\left[\left[X_{1}, \ldots, X_{n}\right]\right]$ is a $\pi$-domain for every integer $n \geq 1$, then $R[[\mathcal{X}]]_{3}$ is a $\pi$-domain.

Proof. Let $\delta$ be a nonunit element of $R[[\mathcal{X}]]_{3}$. By Proposition 4.3 , there exists a finite subset $\mathcal{F}$, depending on $\delta$, of $\mathcal{X}$ such that for every finite subset $\mathcal{K} \supseteq \mathcal{F}$ of $\mathcal{X}$,

$$
(\mathcal{F}(\delta))=P_{1, \mathcal{F}}^{m_{1}} \cdots P_{n, \mathcal{F}}^{m_{n}} \quad \text { and } \quad(\mathcal{K}(\delta))=P_{1, \mathcal{K}}^{m_{1}} \cdots P_{n, \mathcal{K}}^{m_{n}},
$$

where $P_{i, \mathcal{F}}$ 's and $P_{i, \mathcal{K}}$ 's are distinct prime ideals of $R[[\mathcal{F}]]$ and $R[[\mathcal{K}]]$, respectively. Moreover, $\left\{P_{i, \mathcal{K}}\right\}_{\mathcal{K} \supseteq \mathcal{F}}$ is a projective sequence of invertible prime ideals for each $i$. Put $P_{i, \infty}=\left\{\alpha \in R[[\mathcal{X}]]_{3} \mid \mathcal{K}(\alpha) \in P_{i, \mathcal{K}}\right.$ for every finite $\left.\mathcal{K} \supseteq \mathcal{F}\right\}$. Note that $\mathcal{K}\left(P_{i, \infty}\right) \subseteq P_{i, \mathcal{K}}$ for every finite subset $\mathcal{K} \supseteq \mathcal{F}$ of $\mathcal{X}$. Since $\delta \in P_{i, \infty}$, it follows from Lemma 4.4 that $P_{i, \infty}$ is a prime ideal of $R[[\mathcal{X}]]_{3}$ containing $\delta$.

We claim that $(\delta)=P_{1, \infty}^{m_{1}} \cdots P_{n, \infty}^{m_{n}}$.

Let $x \in P_{1, \infty}^{m_{1}} \cdots P_{n, \infty}^{m_{n}}$. Since $\mathcal{K}\left(P_{i, \infty}\right) \subseteq P_{i, \mathcal{K}}$ for every finite subset $\mathcal{K} \supseteq \mathcal{F}$ of $\mathcal{X}$ and each $i$, it follows from Theorem 2.1.7) that

$$
\mathcal{K}(x) \in \mathcal{K}\left(P_{1, \infty}^{m_{1}} \cdots P_{n, \infty}^{m_{n}}\right) \subseteq P_{1, \mathcal{K}}^{m_{1}} \cdots P_{n, \mathcal{K}}^{m_{n}}=(\mathcal{K}(\delta)) .
$$

Thus $\mathcal{K}(x)=\mathcal{K}(\delta) \gamma_{\mathcal{K}}$ for some $\gamma_{\mathcal{K}} \in R[[\mathcal{K}]]$. For any pair of finite subsets $\mathcal{K}$ and $\mathcal{L}$ of $\mathcal{X}$ such that $\mathcal{F} \subseteq \mathcal{K} \subseteq \mathcal{L}$, we have $\mathcal{L}(x)=\mathcal{L}(\delta) \gamma_{\mathcal{L}}$ and $\mathcal{K}(x)=\mathcal{K}(\delta) \gamma_{\mathcal{K}}$. Since $\mathcal{K}(\mathcal{L}(x))=$ $\mathcal{K}(x)$ and $\mathcal{K}(\mathcal{L}(\delta))=\mathcal{K}(\delta)$, we have $\mathcal{K}\left(\gamma_{\mathcal{L}}\right)=\gamma_{\mathcal{K}}$. Therefore, $\left\{\gamma_{\mathcal{K}}\right\}_{\mathcal{K} \supseteq \mathcal{F}}$ is a projective sequence. In view of Theorem 2.1 (5), there exists $\gamma \in R[[\mathcal{X}]]_{3}$ such that $\mathcal{K}(\gamma)=\gamma_{\mathcal{K}}$ for every finite subset $\mathcal{K} \supseteq \mathcal{F}$ of $\mathcal{X}$. Then $\mathcal{K}(x)=\mathcal{K}(\delta) \gamma_{\mathcal{K}}=\mathcal{K}(\delta) \mathcal{K}(\gamma)=\mathcal{K}(\delta \gamma)$ for every finite subset $\mathcal{K} \supseteq \mathcal{F}$ of $\mathcal{X}$. It follows from Theorem 2.1 4 ) that $x=\delta \gamma$. Therefore $P_{1, \infty}^{m_{1}} \cdots P_{n, \infty}^{m_{n}} \subseteq(\delta)$.

It follows from the remark just after Proposition 4.3 that there exist invertible ideals $Q_{i}$ in $R[[\mathcal{X}]]_{3}$ such that $\mathcal{K}\left(Q_{i}\right)=P_{i, \mathcal{K}}$ for every finite subset $\mathcal{K} \supseteq \mathcal{F}$ of $\mathcal{X}$ and each $i$. Note that $Q_{i} \subseteq P_{i, \infty}$ for each $i$. Since $\mathcal{K}\left(Q_{i}\right)=P_{i, \mathcal{K}}$ for every finite subset $\mathcal{K} \supseteq \mathcal{F}$ of $\mathcal{X}$, we have

$$
\left\{\begin{array}{l}
Q_{1}^{m_{1}} \cdots Q_{n}^{m_{n}} \subseteq P_{1, \infty}^{m_{1}} \cdots P_{n, \infty}^{m_{n}} \subseteq(\delta) \\
\mathcal{K}\left(Q_{1}^{m_{1}} \cdots Q_{n}^{m_{n}}\right)=\mathcal{K}\left(Q_{1}\right)^{m_{1}} \cdots \mathcal{K}\left(Q_{n}\right)^{m_{n}}=P_{1, \mathcal{K}}^{m_{1}} \cdots P_{n, \mathcal{K}}^{m_{n}}=(\mathcal{K}(\delta)) .
\end{array}\right.
$$

Thus $Q_{1}^{m_{1}} \ldots Q_{n}^{m_{n}} \subseteq(\delta)$ and their $\mathcal{K}$-projections are the same. Therefore, by Theorem 3.5. $Q_{1}^{m_{1}} \cdots Q_{n}^{m_{n}}=(\delta)$ and hence $(\delta)=P_{1, \infty}^{m_{1}} \cdots P_{n, \infty}^{m_{n}}$.

Remark. Since $P_{i, \infty}$ is an invertible ideal of $R[[\mathcal{X}]]_{3}$ containing $Q_{i}$ and $\mathcal{K}\left(P_{i, \infty}\right)=$ $\mathcal{K}\left(Q_{i}\right)$ for a finite subset $\mathcal{K}$, it follows from Theorem 3.5 that $P_{i, \infty}=Q_{i}=f P_{i}^{\prime}[[\mathcal{X}]]_{3}$ in $R[[\mathcal{X}]]_{3}$, where $f \in K[[\mathcal{X}]]_{3}$ and $P_{i}^{\prime}$ is an invertible ideal of $R$.

If $R$ is a Noetherian regular domain, then $R\left[\left[X_{1}, \ldots, X_{n}\right]\right]$ is also a Noetherian regular domain. Since a Noetherian regular domain is a $\pi$-domain, $R$ is a formally stable $\pi$ domain. In particular, if $R$ is a Dedekind domain, then $R$ is a formally stable $\pi$-domain. The following is an immediate application of Theorem 4.5 . 
Corollary 4.6. Let $R$ be an integral domain and $\mathcal{X}$ be a set of indeterminates over $R$.

(i) If $R$ is a Noetherian regular domain, then $R[[\mathcal{X}]]_{3}$ is a $\pi$-domain.

(ii) If $R$ is a Dedekind domain, then $R[[\mathcal{X}]]_{3}$ is a $\pi$-domain.

In [6], Claborn showed that if $R$ is a Noetherian regular domain, then $\mathrm{Cl}(R) \cong$ $\mathrm{Cl}\left(R\left[\left[X_{1}, \ldots, X_{n}\right]\right]\right)$ canonically and $\mathrm{Cl}\left(R\left[\left[X_{1}, \ldots, X_{n}\right]\right]_{R^{*}}\right)=0$, where $R^{*}$ is the set of nonzero elements of $R$. Since a regular domain is a $\pi$-domain, our next result is a generalization of Claborn's results to a formally stable $\pi$-domain as well as to an arbitrary set of indeterminates.

Theorem 4.7. If $R$ is a formally stable $\pi$-domain and $\mathcal{X}$ is a set of indeterminates over $R$, then

(i) $\mathrm{Cl}(R) \cong \mathrm{Cl}\left(R[[\mathcal{X}]]_{3}\right)$ canonically,

(ii) $\mathrm{Cl}\left(\left(R[[\mathcal{X}]]_{3}\right)_{R^{*}}\right)=0$; thus $\left(R[[\mathcal{X}]]_{3}\right)_{R^{*}}$ is a UFD.

Proof. (i) By Theorem $4.5, R[[\mathcal{X}]]_{3}$ is a $\pi$-domain. By Theorem 2.2 6), every divisorial ideal of $R[[\mathcal{X}]]_{3}$ is invertible. Since $\mathrm{Cl}\left(R[[\mathcal{X}]]_{3}\right)=\operatorname{Pic}\left(R[[\mathcal{X}]]_{3}\right)$, it follows from Corollary 3.12 that $\mathrm{Cl}(R) \cong \mathrm{Cl}\left(R[[\mathcal{X}]]_{3}\right)$.

(ii) Let $J$ be a divisorial ideal of $\left(R[[\mathcal{X}]]_{3}\right)_{R^{*}}$. It is easy to see that $J=I_{R^{*}}$, where $I$ is a divisorial ideal of $R[[\mathcal{X}]]_{3}[12]$. By Theorem 3.11, $I=f I^{\prime}[[\mathcal{X}]]_{3}$, where $I^{\prime}$ is an invertible ideal of $R$. Therefore, $I_{R^{*}}=\left(f I^{\prime}[[\mathcal{X}]]_{3}\right) R_{R^{*}}=f\left(R[[\mathcal{X}]]_{3}\right)_{R^{*}}$. Thus $J=I_{R^{*}}$ is a principal ideal.

Corollary 4.8. If $R$ is a Noetherian regular domain and $\mathcal{X}$ is a set of indeterminates over $R$, then

(i) $\mathrm{Cl}(R) \cong \mathrm{Cl}\left(R[[\mathcal{X}]]_{3}\right)$ canonically,

(ii) $\mathrm{Cl}\left(\left(R[[\mathcal{X}]]_{3}\right)_{R^{*}}\right)=0$; thus $\left(R[[\mathcal{X}]]_{3}\right)_{R^{*}}$ is a UFD.

\section{Extension to rings with zero-divisors}

Let $R$ be a commutative ring with identity. We use the notation $\operatorname{dim} R$ for the Krull dimension of $R . R$ is called a $\pi$-ring if every principal ideal is a product of prime ideals. If $R\left[\left[X_{1}, \ldots, X_{n}\right]\right]$ is a $\pi$-ring for each finite set $\left\{X_{1}, \ldots, X_{n}\right\}$ of indeterminates over $R$, then $R$ is called a formally stable $\pi$-ring. Let $\mathcal{X}$ be a set of indeterminates over $R$. In this section, we will prove that if $R$ is a formally stable $\pi$-ring, then $R[[\mathcal{X}]]_{3}$ is also a $\pi$-ring. As a corollary, it will follow that $R\left[[\mathcal{X}]_{3}\right.$ is a $\pi$-ring if $R$ is a Noetherian regular ring. We abbreviate a Noetherian regular ring as a regular ring.

A special primary ring $R$ is a quasi-local ring with maximal ideal $M$ such that each proper ideal of $R$ is a power of $M$. It is clear that if $R$ is a special primary ring, then $\operatorname{dim} R=0$.

Theorem 5.1 ([11]). A quasi-local $\pi$-ring $R$ with dimension greater than zero is an integral domain. 
Lemma 5.2 ([11]). If $\left\{R_{i}\right\}_{i=1}^{n}$ is a finite set of ideals of $R$ such that $R=R_{1} \oplus \cdots \oplus R_{n}$, then $R$ is a $\pi$-ring if and only if each $R_{i}$ is a $\pi$-ring.

Theorem 5.3 ([11, 15]). $R$ is a $\pi$-ring if and only if $R$ is a finite direct sum of $\pi$-domains and special primary rings.

Theorem 5.4. If $R$ is a formally stable $\pi$-ring, then $R[[\mathcal{X}]]_{3}$ is a $\pi$-ring.

Proof. Replacing $R$ by $R[[X]]$, we may assume that $R$ is a $\pi$-ring. By Theorem $5.3, R$ can be written as

$$
R=R_{1} \oplus \cdots \oplus R_{n},
$$

where $R_{i}$ is either a $\pi$-domain or a special primary ring. A special primary ring is clearly a $\pi$-ring. So each $R_{i}$ is a $\pi$-ring. Since $R$ is a formally stable $\pi$-ring, $R_{1}[[X]] \oplus \cdots \oplus$ $R_{n}[[X]]=R[[X]]$ is a $\pi$-ring. By Lemma 5.2, each $R_{i}[[X]]$ is a $\pi$-ring. We will show that each $R_{i}$ is in fact a $\pi$-domain. Assume that $R_{i}$ is a special primary ring. Since $R_{i}[[X]]$ is a quasi-local $\pi$-ring and $\operatorname{dim} R_{i}[[X]] \geq 1, R_{i}[[X]]$ is an integral domain by Theorem 5.1 and hence $R_{i}$ is an integral domain. So $R_{i}$ is a $\pi$-domain.

Now we have $R=R_{1} \oplus \cdots \oplus R_{n}$ and each $R_{i}$ is a $\pi$-domain. Since $R$ is a formally stable $\pi$-ring, $\bigoplus_{i=1}^{n} R_{i}\left[\left[X_{1}, \ldots, X_{m}\right]\right]=R\left[\left[X_{1}, \ldots, X_{m}\right]\right]$ is a $\pi$-ring for each $m \geq 1$. By Lemma 5.2, each $R_{i}\left[\left[X_{1}, \ldots, X_{m}\right]\right]$ is a $\pi$-domain. By Theorem 4.5, each $R_{i}[[\mathcal{X}]]_{3}$ is a $\pi$-domain. Therefore, by Theorem 5.3, $R[[\mathcal{X}]]_{3}=R_{1}[[\mathcal{X}]]_{3} \oplus \cdots \oplus R_{n}[[\mathcal{X}]]_{3}$ is a $\pi$-ring.

It is well known that every regular ring is a finite direct sum of regular domains. Since a regular domain is a $\pi$-domain, a regular ring is a $\pi$-ring by Theorem 5.3 . Since $R[[X]]$ is also a regular ring if $R$ is a regular ring, a regular ring is a formally stable $\pi$-ring.

Corollary 5.5. If $R$ is a Noetherian regular ring and $\mathcal{X}$ is a set of indeterminates over $R$, then $R[[\mathcal{X}]]_{3}$ is a $\pi$-ring.

Acknowledgments. The first author was supported by the Korea Research Foundation Grant (KRF2008-041-C00003) funded by the Korean Government (MOEHRD, Basic Research Promotion Fund).

\section{References}

[1] Anderson, D. D.: $\pi$-domains, overrings, and divisorial ideals. Glasgow Math. J. 19, 199-203 (1978) Zbl 0379.13011 MR 0485845

[2] Anderson, D. D.: Globalization of some local properties in Krull domains. Proc. Amer. Math. Soc. 85, 141-145 (1982) Zbl 0498.13009 MR 0652428

[3] Auslander, M., Buchsbaum, D.: Unique factorization in regular local rings. Proc. Nat. Acad. Sci. USA 45, 733-734 (1959) Zbl 0084.26504 MR 0103906

[4] Brewer, J. W.: Power Series over Commutative Rings. Lecture Notes in Pure Appl. Math. 64, Dekker, New York (1981). Zbl 0476.13015 MR 0612477

[5] Cashwell, E. D., Everett, C. J.: Formal power series. Pacific J. Math. 13, 45-64 (1963) Zbl 0117.02603 MR 0155852 
[6] Claborn, L.: Note generalizing a result of Samuel's, Pacific J. Math. 15, 805-808 (1965) Zbl 0135.08004 MR 0188248

[7] Deckard, D.: M. A. Thesis. Rice Univ. (1961)

[8] Deckard, D., Durst, L. K.: Unique factorization in power series rings and semigroups, Pacific J. Math. 16, 239-242 (1966) Zbl 0136.31801 MR 0184968

[9] Fossum, R.: The Divisor Class Group of a Krull Domain. Springer, New York (1973) Zbl 0256.13001 MR 0382254

[10] Gilmer, R.: Power series rings over a Krull domain. Pacific J. Math. 13, 543-550 (1969) Zbl 0179.34502 MR 0245571

[11] Gilmer, R.: Multiplicative Ideal Theory. Dekker, New York (1972) Zbl 0248.13001 MR 0427289

[12] Kang, B. G.: On the converse of a well-known fact about Krull domains. J. Algebra 124, 284-299 (1989) Zbl 0694.13011 MR 1011595

[13] Kaplansky, I.: Commutative Rings. Rev. ed., Univ. of Chicago Press, Chicago (1974) Zbl 0296.13001 MR 0345945

[14] Levitz, K.: A characterization of general Z.P.I.-rings. Proc. Amer. Math. Soc. 13, 376-380 (1972) Zbl 0212.38401 MR 0294312

[15] Mori, S.: Über die Produktzerlegung der Hauptideale III. J. Sci. Hiroshima Univ. (A) 10, 85-94 (1940) Zbl 0023.19903 MR 0001965

[16] Nishimura, H.: On the unique factorization theorem for formal power series. J. Math. Kyoto Univ. 7, 151-160 (1967) Zbl 0159.33401 MR 0225766

[17] Samuel, P.: On unique factorization domains. Illinois J. Math. 5, 1-17 (1961) Zbl 0147.29202 MR 0121382 